\title{
Shear wave elastography of the radial nerve in healthy subjects
}

Journal of International Medical Research 49(I) 1-9

(C) The Author(s) 2021

Article reuse guidelines: sagepub.com/journals-permissions DOI: I0.I I 77/0300060520987938 journals.sagepub.com/home/imr

(SAGE

\author{
Mohamed A Bedewi' (D, Mamdouh A Kotb ${ }^{2,3}$, \\ Nasser M Aldossary', Ahmed M Abodonya ${ }^{4,5}$, \\ Ayman K Saleh ${ }^{5,6}$ and \\ Sherine Mohamed Swify ${ }^{7}$
}

\begin{abstract}
Objective: This study was performed to examine the sonoelastographic features of the radial nerve in healthy subjects.

Methods: In this observational cross-sectional study, shear wave elastography was used to evaluate the radial nerve. The cross-sectional area and stiffness were measured.

Results: The study included 37 nerves in 20 healthy adult subjects. The mean cross-sectional area of the radial nerve at the arm was $6.1 \mathrm{~mm}^{2}$. The mean stiffness of the radial nerve in the short axis was $30.3 \mathrm{kPa}$, and that in the long axis was $34.9 \mathrm{kPa}$. The elasticity measurements were significantly different between the long axis and short axis.

Conclusion: The elastic modulus of the radial nerve was studied in healthy subjects and can serve as a reference for future assessment of different radial nerve pathologies.
\end{abstract}

\section{Keywords}

Radial, nerve, ultrasound, elastography, shear wave, neuropathy

Date received: 13 August 2020; accepted: II December 2020

\footnotetext{
'Department of Internal Medicine, College of Medicine, Prince Sattam Bin Abdulaziz University, Al-Kharj, Kingdom of Saudi Arabia

${ }^{2}$ Neurology Department, College of Medicine, Prince Sattam Bin Abdulaziz University, Al-Kharj, Kingdom of Saudi Arabia

${ }^{3}$ Neurology Department, Faculty of Medicine, Minia University, Minia, Egypt
}

\footnotetext{
${ }^{4}$ Anesthesia and Intensive Care Department, Faculty of Medicine, Al-Azhar University, Cairo, Egypt

${ }^{5}$ Surgery Department, College of Medicine, Prince Sattam bin Abdulaziz University, Kingdom of Saudi Arabia

${ }^{6}$ Orthopedic Department, Faculty of Medicine for Girls, Alazhar University, Cairo

${ }^{7}$ Ministry of Health, Alexandria, Egypt

Corresponding author:

Mohamed A Bedewi, Department of Internal Medicine,

College of Medicine, Prince Sattam Bin Abdulaziz

University, PO Box 173, Al-kharj I 1942, Kingdom of Saudi Arabia.

Email: mohamedbedewi@yahoo.com
} 


\section{Introduction}

The radial nerve is a major nerve of the upper limb. ${ }^{1}$ The radial nerve forms as a continuation of the posterior cord of the brachial plexus and is derived from the ventral roots of spinal nerves $\mathrm{C} 5$ to $\mathrm{T} 1$. It then arises at the axilla and enters the posterior compartment of the arm. The nerve travels in the spiral groove of the humerus and then wraps around it laterally. At the level of the lateral epicondyle, the radial nerve branches into a deep motor branch and a superficial sensory branch. ${ }^{2}$ The radial nerve provides motor supply to the posterior compartment of the arm, posterior forearm compartment, and extensor muscles of the wrist and fingers. It also provides sensory supply to part of the anterolateral arm, distal posterior arm, posterior forearm, lateral half of the little finger, middle finger, index finger, and posterior aspect of the thumb. ${ }^{1}$ Injury to the radial nerve can lead to motor consequences such as the inability to extend the wrist, fingers, and arm in addition to paresthesia about the sensory distribution.

Surgery/trauma near the radial nerve is associated with a risk of injury. Radial neuropathy can result from ischemia, compression, arm fractures, and penetrating wounds. These injuries commonly present with wrist drop. The severity of the neuropathy is determined by the level of injury. ${ }^{2}$ The radial nerve can be compressed at several sites, and the clinical syndrome is dependent upon the site of compression. ${ }^{1}$ A common cause of injury to the radial nerve is humeral shaft fracture. Primary injury results from stretching or crushing of the nerve at the time of fracture, whereas secondary injury is associated with surgery during open reduction and fixation. Scar tissue can lead to tertiary injury by limiting fascial gliding and causing nerve entrapment, encasement, and sometimes adhesions. ${ }^{3}$ Radial nerve block is indicated in various upper limb surgical procedures and for patients with pain syndromes and postoperative pain. The radial nerve block procedure is performed using ultrasound (US) guidance. ${ }^{4}$

The diagnosis of radial neuropathy/ entrapment is primarily based on the physical examination findings, clinical history, and electrodiagnostic test results. In addition to magnetic resonance imaging, US was introduced to play a complimentary role in the evaluation of peripheral nerve pathologies. The nerve size can be estimated by measuring the cross-sectional area (CSA) during US examinations. Nerve fascicles and nerve echogenicity can also be assessed by US. Elastography is a relatively new technique that quantifies tissue elasticity in response to applied force. It can also be used to evaluate the mechanical properties of tissues. ${ }^{5-9}$ Shear wave elastography (SWE) is one of two main types of elastography used to evaluate the neuromuscular system. In the first type (strain elastography), mild probe compression causes tissue displacement. This allows for a colored, semiquantitative, and scaled qualitative evaluation of elasticity. The second type is SWE, in which the probe produces a pulse that propagates through the tissue of interest, producing waves in a shear manner. SWE produces quantitative results in kilopascals ( $\mathrm{kPa}$, Young's modulus), is less operator-dependent, and is more reproducible. Major peripheral nerves such as the median, sciatic, and tibial nerves have been studied by SWE, especially in patients with peripheral neuropathy. ${ }^{5-16}$ The present study was performed to examine the sonoelastographic features of the radial nerve in healthy subjects.

\section{Methods}

\section{Participants}

In this observational cross-sectional study, 37 radial nerves of 20 healthy adult subjects 
were evaluated. The participants were recruited from March 2020 to May 2020. The inclusion criteria were a clinically healthy condition, male or female sex, and age range of 25 to 46 years. The exclusion criteria were peripheral neuropathy, a history of numbness or upper limb pain, weakness or paresthesia, and injury to the upper limb. Each participant's sex, age, weight, body mass index (BMI), and height were recorded. All subjects enrolled in this study were free from any diseases related to the neuromuscular system as indicated by clinical examination and electrophysiologic methods.

Institutional review board approval (PSAU/COM/RC/IRB/p/70) was obtained before commencement of the study, and all participants provided written informed consent.

Technique. The US examination was performed with a 4- to $18-\mathrm{MHz}$ linear-array transducer (L18-4 transducer, EPIQ Elite SW 5.0.1 US System; Philips, Bothell, WA, USA). A radiologist (M.B.) with 19 years of experience performed all examinations, and the images were reviewed by a neurologist (M.K). All participants were examined in the sitting position, with the participant resting his or her arm on the thigh and the wrist supinated. The radial nerve was identified at the mid-arm level. The CSA was measured in $\mathrm{mm}^{2}$. For the SWE measurements, each subject was scanned three times with removal of the probe from the skin between measurements. To increase the reliability of the reported stiffness values, a confidence map was used to mask areas below a specific confidence level. A large amount of gel was used with a light touch of the probe to decrease the pressure effect on the skin. The radial nerve was first identified in the short axis and SWE measurements were taken, and the probe was then rotated 90 degrees to acquire longitudinal SWE measurements.
After identifying the nerve in each examination, the probe was held stationary for 3 to $4 \mathrm{~s}$, and a 2-mm-diameter circular region of interest was placed within the hyperechoic epineurium. After viewing the color map, real-time shear wave images were recorded with color coding. The readings consisted of the median, maximum, and average elasticity with standard deviation and were reported in $\mathrm{kPa}$. The color scale was mapped to a 0 - to $200-\mathrm{kPa}$ range. The spectrum of scale colors ranged from blue for softer tissues through red for stiffer tissues (Figures 1 and 2).

\section{Electrophysiologic methods}

The nerve conduction studies were performed with a Neuropack device (Nihon Kohden, Tokyo, Japan). All studies were performed under a standard room temperature of $25^{\circ} \mathrm{C}$. Hand temperature was maintained at $\geq 32^{\circ} \mathrm{C}$. Electrodiagnostic studies were performed on both hands and feet in all subjects by an expert neurologist.

\section{Statistical analysis}

Statistical analysis was performed using IBM SPSS Statistics for Windows, Version 21.0 (IBM Corp., Armonk, NY, USA). Data are presented as mean \pm standard deviation and range. Intraobserver variability was measured using Kohen's kappa test. An independent-samples test was used to assess the difference in the mean elasticity between the right and left radial nerves. The correlations between the mean elasticity bilaterally and age, weight, height, and BMI were calculated by Pearson's correlation coefficient test. Statistical significance was defined as $\mathrm{P}<0.05$.

\section{Results}

The study included 37 radial nerves of 20 healthy adult subjects with a mean age of $32.9 \pm 7.2$ years (range, 24-46 years), mean 


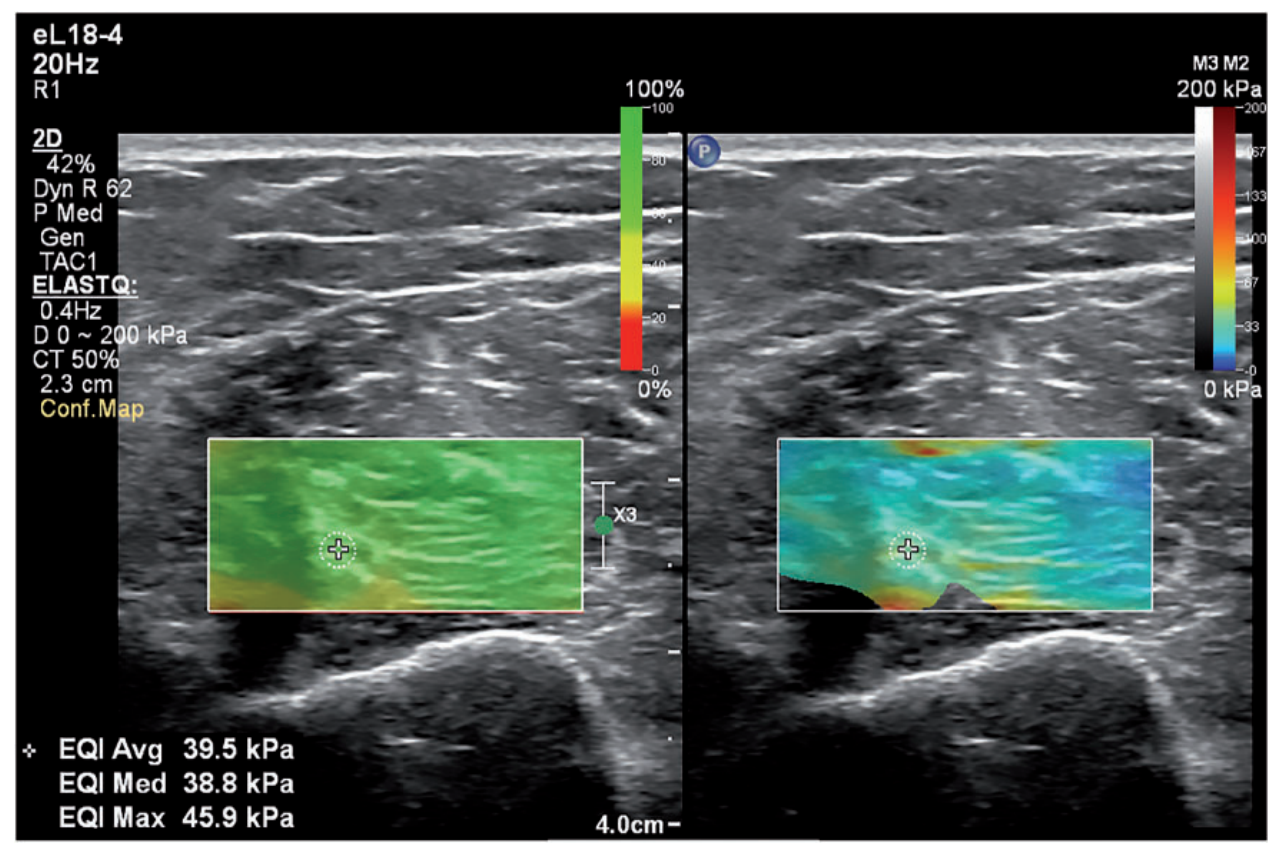

Figure I. Short-axis view of radial nerve shear wave elastography (confidence map on left, color map scale on right) for measurement of stiffness in $\mathrm{kPa}$.

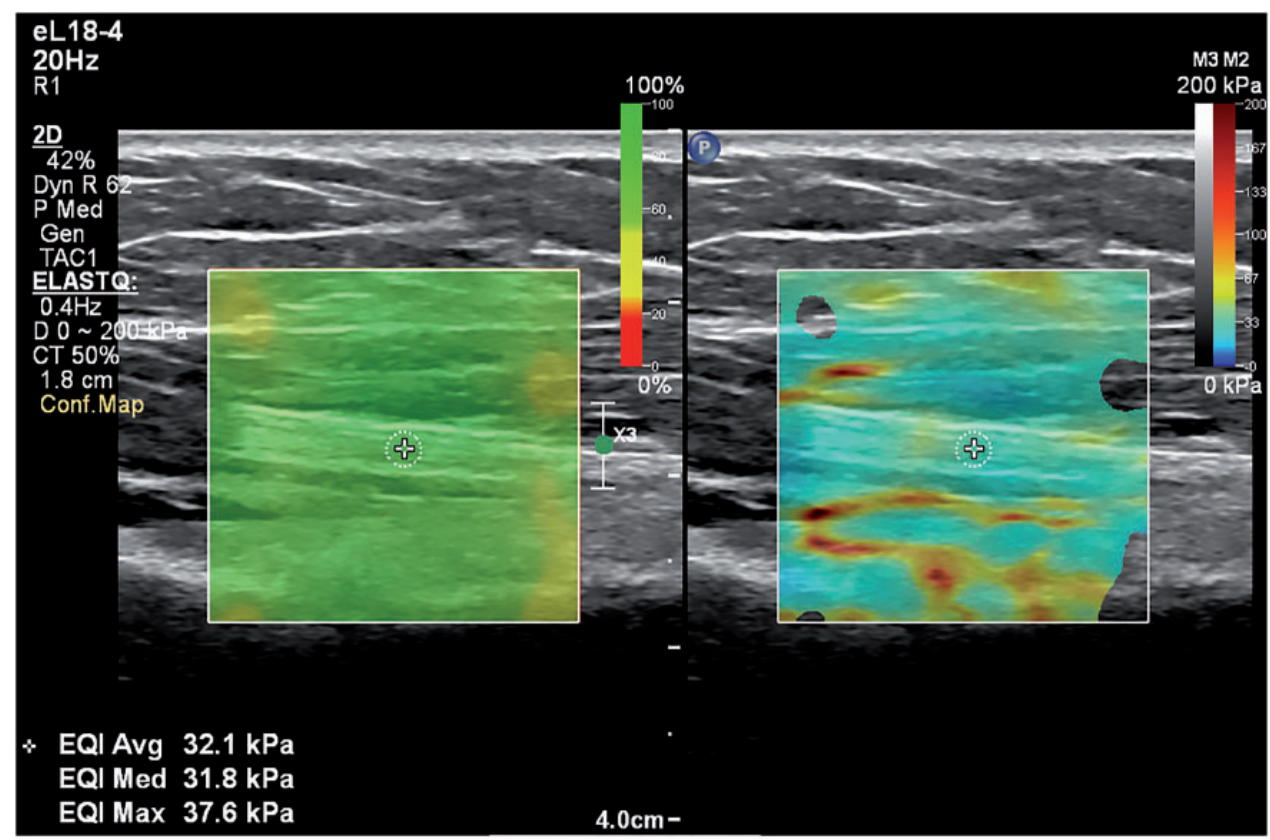

Figure 2. Long-axis view of radial nerve shear wave elastography (confidence map on left, color map scale on right) for measurement of stiffness in $\mathrm{kPa}$. 
height of $158.6 \pm 8.8 \mathrm{~cm}$ (range, 144 $177 \mathrm{~cm})$, mean weight of $60.4 \pm 10.5 \mathrm{~kg}$ (range, $43-84 \mathrm{~kg}$ ), and mean BMI of $23.8 \pm 3.1 \mathrm{~kg} / \mathrm{m}^{2}$ (range, $19.0-30.9 \mathrm{~kg} / \mathrm{m}^{2}$ ). Table 1 shows the demographic characteristics of the study participants.

The mean CSA of the radial nerve at the arm was $6.1 \mathrm{~mm}^{2}$ (range, 3.5-10.6土 $1.7 \mathrm{~mm}^{2}$ ). The mean shear elastic modulus of the nerve in the short axis was $30.3 \mathrm{kPa}$ (range, $19.0-40.9 \pm 6.4 \mathrm{kPa}$ ), and that in the long axis was $34.9 \mathrm{kPa}$ (range, 25.5-45.4 \pm $5.2 \mathrm{kPa}$ ). Table 2 shows the CSA and stiffness values of the nerve. The intraobserver reliability calculations resulted in an overall intraclass correlation coefficient of 0.80 . No statistically significant differences were noted between the right and left sides regarding the CSA, shear wave elastic modulus of the nerve in the short axis, or shear wave elastic modulus of the radial nerve in the long axis. A negative correlation was noted between age and the long-axis shear modulus $(\mathrm{P}=0.018)$. A positive correlation

Table I. Demographic characteristics of study participants.

\begin{tabular}{ll}
\hline Age, years & $32.9 \pm 7.2$ \\
\hline Sex & \\
Male & $6(30)$ \\
Female & $14(70)$ \\
Height, cm & $158.6 \pm 8.8$ \\
Weight, kg & $60.4 \pm 10.5$ \\
Body mass index, $\mathrm{kg} / \mathrm{m}^{2}$ & $23.8 \pm 3.1$ \\
\hline
\end{tabular}

Data are presented as mean \pm standard deviation or $\mathrm{n}(\%)$.

Table 2. CSA and stiffness values of the radial nerve.

\begin{tabular}{lll}
\hline & & $P$ \\
\hline Radial CSA, mm & $6.1 \pm 1.7(3.5-10.6)$ & 0.447 \\
Radial SA, kPa & $30.3 \pm 6.4(19.0-47.9)$ & 0.100 \\
Radial LA, kPa & $34.9 \pm 5.2(25.5-45.4)$ & 0.251 \\
\hline
\end{tabular}

Data are presented as mean \pm standard deviation (range). CSA, cross-sectional area; SA, short axis; LA, long axis. was noted between the BMI and short-axis shear modulus $(\mathrm{P}=0.039)$. No significant correlation was noted between the shear modulus and any other demographic factors. The elasticity measurements were significantly different between the long axis and short axis $(\mathrm{P}=0.001)$. The radial nerve elastic modulus showed no correlation with the CSA in either the long or short axis. Table 3 shows the correlations between the CSA, stiffness, and demographic characteristics in our study. Table 4 shows the results of the nerve conduction studies.

\section{Discussion}

We studied the radial nerve at the arm in healthy adult subjects by SWE. The relationships between elasticity and height, weight, BMI, and sex were also studied. The mean CSA of the radial nerve in our study was $6.1 \mathrm{~mm}^{2}$ (range, 3.5-10.6土 $1.7 \mathrm{~mm}^{2}$ ). This is comparable with the findings reported by Cartwright et al. ${ }^{17}$ (7.9 \pm $\left.2.7 \mathrm{~mm}^{2}\right)$, Qrimli et al. ${ }^{18}\left(6.5 \mathrm{~mm}^{2}\right)$, and Bedewi et al. ${ }^{19}\left(5.5 \pm 1.9 \mathrm{~mm}^{2}\right)$. Several studies have used SWE to evaluate important upper limb nerves. Paluch et al. ${ }^{20}$ reported a mean stiffness of $33.1 \pm$ $10.13 \mathrm{kPa}$ (range, $19-51 \mathrm{kPa}$ ) for the ulnar nerve at the level of the cubital tunnel. The same authors reported higher stiffness values of the ulnar nerve at the forearm (49 $\mathrm{kPa}$; range, $23-68 \mathrm{kPa}) .^{21}$ Cornelson et al. $^{22}$ reported a mean stiffness of $13.2 \pm$ $11.26 \mathrm{kPa}$ for the ulnar nerve at the cubital tunnel. Kantarci et al. ${ }^{23}$ used SWE to study the median nerve proximal to the carpal tunnel and revealed a mean stiffness of $32 \mathrm{kPa}$. Some authors have reported difficulty in obtaining reliable results when measurements were obtained in the short axis. Aslan et al. ${ }^{24}$ reported higher stiffness values in the long axis than in the short axis. In our study, we performed SWE measurements in both axes and found a statistically 
Table 3. Correlations among demographic factors, CSA, and stiffness in long and short axes.

\begin{tabular}{|c|c|c|c|}
\hline & Radial CSA & Radial SA & Radial LA \\
\hline \multicolumn{4}{|l|}{ Age } \\
\hline Pearson correlation & -0.223 & 0.077 & $-0.386 *$ \\
\hline Sig. (two-tailed) & 0.185 & 0.651 & 0.018 \\
\hline $\mathrm{n}$ & 37 & 37 & 37 \\
\hline \multicolumn{4}{|l|}{ Weight } \\
\hline Pearson correlation & -0.100 & 0.239 & -0.090 \\
\hline Sig. (two-tailed) & 0.556 & 0.155 & 0.596 \\
\hline $\mathrm{n}$ & 37 & 37 & 37 \\
\hline \multicolumn{4}{|l|}{ Height } \\
\hline Pearson correlation & 0.072 & 0.051 & -0.028 \\
\hline Sig. (two-tailed) & 0.671 & 0.762 & 0.871 \\
\hline $\mathrm{n}$ & 37 & 37 & 37 \\
\hline \multicolumn{4}{|l|}{ BMI } \\
\hline Pearson correlation & -0.199 & $0.34 I^{*}$ & -0.079 \\
\hline Sig. (two-tailed) & 0.238 & 0.039 & 0.644 \\
\hline $\mathrm{n}$ & 37 & 37 & 37 \\
\hline \multicolumn{4}{|l|}{ Radial CSA } \\
\hline Pearson correlation & I & -0.002 & 0.006 \\
\hline Sig. (two-tailed) & & 0.992 & 0.974 \\
\hline n & 37 & 37 & 37 \\
\hline \multicolumn{4}{|l|}{ Radial SA } \\
\hline Pearson correlation & -0.002 & 1 & 0.168 \\
\hline Sig. (two-tailed) & 0.992 & & 0.321 \\
\hline $\mathrm{n}$ & 37 & 37 & 37 \\
\hline \multicolumn{4}{|l|}{ Radial LA } \\
\hline Pearson correlation & 0.006 & 0.168 & I \\
\hline Sig. (two-tailed) & 0.974 & 0.321 & \\
\hline $\mathrm{n}$ & 37 & 37 & 37 \\
\hline
\end{tabular}

*Correlation is significant at the 0.05 level (two-tailed).

CSA, cross-sectional area; SD, standard deviation; SA, short axis; LA, long axis; BMI, body mass index.

significant difference between the elasticity in the short axis $(30.3 \mathrm{kPa})$ and long axis $(34.9 \mathrm{kPa})$. This difference might be attributed to the relative proximity of the radial nerve at this site to the humerus.

Battaglia et al. ${ }^{25}$ studied a single case of benign peripheral nerve schwannoma of the radial nerve and found that the elasticity ranged from 24 to $30 \mathrm{kPa}$ by SWE, coinciding with its soft consistency and benign nature. The authors referred to the reference values of other musculoskeletal structures to indicate the benign nature of the lesion. Su et al. $^{3}$ used SWE to identify sites of neural entrapment by scar tissue and guide perineural hydrodissection in complicated postoperative cases. Such cases are characterized by distortion of the perineural tissues, scar formation, and sometimes adhesion, making identification of the nerve difficult with conventional US. SWE can guide perineural hydrodissection and identify hardened areas. This can avoid unnecessary treatment of nerve segments that appear to be abnormal on conventional US but are not surrounded by scar tissue and therefore do not contribute to symptoms. $^{3} \mathrm{Li}$ and Snedeker $^{26}$ suggested a 
Table 4. Radial nerve conduction studies $(n=40)$.

\begin{tabular}{ll}
\hline SNAP amplitude, $\mu \mathrm{V}$ & $26.45 \pm 1.95$ \\
Sensory conduction velocity, m/s & $54.85 \pm 2.97$ \\
Sensory peak latency, ms & $2.16 \pm 0.189$ \\
Distal motor latency, ms & $2.08 \pm 0.156$ \\
cMAP amplitude, $\mathrm{mV}$ & $5.98 \pm 0.68$ \\
Motor conduction velocity, m/s & $53.6 \pm 2.64$ \\
\hline
\end{tabular}

Data are presented as mean \pm standard deviation. SNAP, sensory nerve action potential; cMAP, compound muscle action potential.

future role of SWE in predicting the risk of injury and tracking the progress of healing. The negative correlation between age and the long-axis shear modulus in our study raises the question of decreased elasticity with age. We found a positive relationship between the BMI and the short-axis shear modulus; however, this finding cannot be generalized because of our small sample size. The proximity of the examined nerve to certain anatomical structures such as bone and synovial fluid may lead to unreliable SWE readings. ${ }^{27}$ In our study, we only included measurements at the mid-arm level. Although it has been recommended to include measurements of the radial nerve at the spiral groove of the humerus, another common site of radial nerve injury, we did not consider this second site because of the very close proximity of the nerve to bone. Other factors can also influence the reliability of nerve stiffness measurements. Substantial variability of nerve stiffness with different limb positions has been reported in the upper limb peripheral nerves. This could lead to recording of higher stiffness values in patients with chronically abnormal limb posture (such as patients with muscle contracture and spasticity). ${ }^{28,29}$ Additionally, the use of different machines with variable frequency transducers and acquisition depths may result in different elasticity values of the same organ. ${ }^{30}$

The present study has several limitations. First, the small sample size decreases the statistical significance of our results. Second, the numbers of women and men were not equal. Third, only healthy subjects were involved without inclusion of different radial nerve pathologies. Future studies involving larger sample sizes and an even sex distribution may help to increase the validity of SWE measurements. These studies should include evaluation of elasticity in patients with different types of compressive neuropathy and comparison of these elasticity values to those of the normal radial nerve. We believe that knowledge of the normal elasticity values is necessary to establish cut-off limits for discriminating normal healthy nerves from diseased nerves.

In conclusion, we believe that SWE of the radial nerve may be a useful future tool for studying changes in the stiffness of the radial nerve in different pathologies.

\section{Acknowledgement}

The authors thank the Deanship of Scientific Research at Prince Sattam Bin Abdulaziz University.

\section{Authors' contributions}

Mohamed Bedewi designed the study, conducted the searches, and was the major contributor to drafting, writing, and editing of the manuscript. Mamdouh Kotb assisted in interpretation of the data. Nasser Aldossary supervised the design of the study. Ahmed Abodonya codesigned the study. Ayman Saleh assisted in the supervision of the study. Sherine Swify assisted in the design of the study. All authors read and approved the final manuscript.

\section{Availability of data and materials}

All data generated or analyzed during the study are available from the first and corresponding author.

\section{Declaration of conflicting interest}

The authors declare that there is no conflict of interest. 


\section{Funding}

This research received no specific grant from any funding agency in the public, commercial, or not-for-profit sectors.

\section{ORCID iD}

Mohamed A Bedewi (D) https://orcid.org/00000001-6723-0749

\section{References}

1. Xiao TG and Cartwright MS. Ultrasound in the evaluation of radial neuropathies at the elbow. Front Neurol 2019; 10: 216.

2. Glover NM and Murphy PB. Anatomy, Shoulder and Upper Limb, Radial Nerve. Treasure Island (FL): StatPearls Publishing, 2020. p. 1-3.

3. Su DC, Chang KV and Lam SKH. Shear wave elastography to guide perineural hydrodissection: two case reports. Diagnostics (Basel) 2020; 10: 348.

4. Durrani MI and Dasgupta S. Radial Nerve Block. Treasure Island (FL): StatPearls Publishing, 2020. p. 1-2.

5. Zakrzewski J, Zakrzewska K, Pluta K, et al. Ultrasound elastography in the evaluation of peripheral neuropathies: a systematic review of the literature. Pol J Radiol 2019; 84: e581-e591.

6. Dąbrowska-Thing A, Zakrzewski J, Nowak $\mathrm{O}$, et al. Ultrasound elastography as a potential method to evaluate entrapment neuropathies in elite athletes: a minireview. Pol J Radiol 2019; 84: e625-e629.

7. Winn N, Lalam R and Cassar-Pullicino V. Sonoelastography in the musculoskeletal system: current role and future directions. World J Radiol 2016; 8: 868-879.

8. Wee TC and Simon NG. Ultrasound elastography for the evaluation of peripheral nerves: a systematic review. Muscle Nerve 2019; 60: 501-512.

9. Hobson-Webb LD. Emerging technologies in neuromuscular ultrasound. Muscle Nerve 2020; 61: 719-725.

10. Davis LC, Baumer TG, Bey MJ, et al. Clinical utilization of shear wave elastography in the musculoskeletal system. Ultrasonography 2019; 38: 2-12.
11. Klauser AS, Miyamoto H, Bellmann-Weiler $\mathrm{R}$, et al. Sonoelastography: musculoskeletal applications. Radiology 2014; 272: 622-633.

12. Taljanovic MS, Gimber LH, Becker GW, et al. Shear-wave elastography: basic physics and musculoskeletal applications. Radiographics 2017; 37: 855-870.

13. Ryu $\mathbf{J}$ and Jeong WK. Current status of musculoskeletal application of shear wave elastography. Ultrasonography 2017; 36: 185-197.

14. Chang PH, Chen YJ, Chang KV, et al. Ultrasound measurements of superficial and deep masticatory muscles in various postures: reliability and influencers. $\mathrm{Sci}$ Rep 2020; 10: 14357.

15. Chiu YH, Chang KV, Chen IJ, et al. Utility of sonoelastography for the evaluation of rotator cuff tendon and pertinent disorders: a systematic review and meta-analysis. Eur Radiol 2020; 30: 6663-6672.

16. Lin CP, Chen IJ, Chang KV, et al. Utility of ultrasound elastography in evaluation of carpal tunnel syndrome: a systematic review and meta-analysis. Ultrasound Med Biol 2019; 45: 2855-2865.

17. Cartwright MS, Passmore LV, Yoon JS, et al. Cross-sectional area reference values for nerve ultrasonography. Muscle Nerve 2008; 37: 566-571.

18. Qrimli M, Ebadi H, Breiner A, et al. Reference values for ultrasonography of peripheral nerves. Muscle Nerve 2016; 53: 538-544.

19. Bedewi MA, Abodonya A, Kotb M, et al. Estimation of ultrasound reference values for the upper limb peripheral nerves in adults: a cross-sectional study. Medicine (Baltimore) 2017; 96: e9306.

20. Paluch $Ł$, Noszczyk B, Nitek $\dot{Z}$, et al. Shearwave elastography: a new potential method to diagnose ulnar neuropathy at the elbow. Eur Radiol 2018; 28: 4932-4939.

21. Paluch $Ł$, Noszczyk BH, Walecki J, et al. Shear-wave elastography in the diagnosis of ulnar tunnel syndrome. J Plast Reconstr Aesthet Surg 2018; 71: 1593-1599.

22. Cornelson SM, Sclocco R and Kettner NW. Ulnar nerve instability in the cubital tunnel of asymptomatic volunteers. $J$ Ultrasound 2019; 22: 337-344. 
23. Kantarci F, Ustabasioglu FE, Delil S, et al. Median nerve stiffness measurement by shear wave elastography: a potential sonographic method in the diagnosis of carpal tunnel syndrome. Eur Radiol 2014; 24: 434-440.

24. Aslan M, Aslan A, Emeksiz HC, et al. Assessment of peripheral nerves with shear wave elastography in type 1 diabetic adolescents without diabetic peripheral neuropathy. J Ultrasound Med 2019; 38: 1583-1596.

25. Battaglia PJ, Carbone-Hobbs V, Guebert GM, et al. High-resolution ultrasonography and shear-wave sonoelastography of a cystic radial nerve Schwannoma. J Ultrasound 2017; 20: 261-266.

26. Li Y and Snedeker JG. Elastography: modality-specific approaches, clinical applications, and research horizons. Skelet Radiol 2011; 40: 389-397.
27. Bortolotto C, Turpini E, Felisaz P, et al. Median nerve evaluation by shear wave elastosonography: impact of "bone-proximity" hardening artifacts and inter-observer agreement. J Ultrasound 2017; 20: 293-299.

28. Greening J and Dilley A. Posture-induced changes in peripheral nerve stiffness measured by ultrasound shear-wave elastography. Muscle Nerve 2017; 55: 213-222.

29. Rugel CL, Franz CK and Lee SSM. Influence of limb position on assessment of nerve mechanical properties using shear wave ultrasound elastography. Muscle Nerve 2020; 61: 616-622.

30. Shin HJ, Kim MJ, Kim HY, et al. Comparison of shear wave velocities on ultrasound elastography between different machines, transducers, and acquisition depths: a phantom study. Eur Radiol 2016; 26: 3361-3367. 